\title{
FACTORS CONTRIBUTING TO EARLY BREASTFEEDING CESSATION AMONG CHINESE MOTHERS: AN EXPLORATORY STUDY
}

\section{Authors:}

1. Marie TARRANT, RN MPH PhD, Associate Professor, School of Nursing, University of Hong Kong

2. Joan E. DODGSON, RN MPH PhD FAAN, Associate Professor, College of Nursing \& Health Innovation, Arizona State University, Phoenix AZ

3. Kendra M. WU, BSc MSE MMedSc, PhD Candidate, School of Public Health, University of Hong Kong

\section{Author Addresses:}

Marie Tarrant, School of Nursing 4/F, William M. W. Mong Block

Li Ka Shing Faculty of Medicine

21 Sassoon Road, Hong Kong

Tel: 85228192643

Fax: 85228726079

Email: tarrantm@hku.hk

Joan E. Dodgson, College of Nursing \& Healthcare Innovation

Arizona State University

Room 372, 500 N. 3rd Street

Phoenix, AZ, USA 85004-0698

Tel: 16024960885

Email: jdodgson@asu.edu

Kendra M. Wu, School of Public Health

5/F, William M. W. Mong Block

Li Ka Shing Faculty of Medicine

21 Sassoon Road, Hong Kong

Tel: 85292838791

Email: kendrawu@hku.hk

Conflict of Interest: The authors have no conflicts of interest to disclose. 


\begin{abstract}
Background. Although more than $85 \%$ of all new mothers in Hong Kong now initiate breastfeeding, few exclusively breastfeed and overall duration is short. More than one-third stop breastfeeding within the first month postpartum.

Objective. To explore the breastfeeding experiences of Hong Kong Chinese mothers who prematurely discontinue breastfeeding to identify contributing factors that might be remediated to help women breastfeed longer.
\end{abstract}

Design. Qualitative exploratory study

Methods. In-depth, exploratory interviews were carried out with 24 new mothers who stopped breastfeeding within one month after birth and content analysis was used to analyse the data.

Findings. Five core themes emerged from the data: unnatural expectations, left to figure it out, uncertainty, unfulfilling experiences, and guilt vs. relief. Because breastfeeding is "natural" participants expected that it would come naturally and thus be easy. When breastfeeding did not happen naturally, however, midwives were too busy to provide breastfeeding support and mothers were left to figure it out on their own. Participants also reported difficulty in gauging whether the infant was getting adequate nutrition from their breastmilk. Few participants had positive breastfeeding experiences; while the decision to stop breastfeeding caused guilt for most participants, others expressed relief at stopping breastfeeding.

Key conclusions and implications for practice. Greater postnatal breastfeeding support, both in the hospital and after the mother returns home, would likely increase the mother's confidence and enhance her mothering experience. Further antenatal and postnatal education on the realistic breastfeeding expectations and the amount of breastmilk required by babies is also important. More research is needed to test professional and peer support breastfeeding 
interventions to provide guidance to policy makers on the most effective breastfeeding support strategies.

Keywords: Breastfeeding, postpartum, support, qualitative research, cessation, Chinese mothers 


\section{Introduction}

New mothers are recommended to exclusively breastfeed their babies for at least six months and then continue to breastfeed for up to 12 months of age or beyond while introducing solid foods (American Academy of Pediatrics, 2012, World Health Organization, 2003). In Hong Kong, over $85 \%$ of all new mothers now initiate breastfeeding (Baby Friendly Hospital Initiative Hong Kong Association, 2013). This is higher than both the UK (74\%) (Department of Health, 2013) and the US (76.5\%) (Centers for Disease Control and Prevention, 2013) but lower than Australia (96\%) (Australian Institute of Health and Welfare, 2011), rural China (98.3\%) (Guo et al., 2013) and many Scandinavian countries (>95\%) (Cattaneo et al., 2010). Although the benefits of breastfeeding to both the infant and the mother have been widely recognized (Ip et al., 2007, Kramer and Kakuma, 2004), early cessation of breastfeeding is common in many developed countries and most infants do not receive the recommended six months of exclusive breastfeeding (Organisation for Economic Co-operation and Development, 2009). In Australia, only 70\% and 60\% of infants are still receiving some breastmilk at 4 and 6 months of age, respectively (Australian Institute of Health and Welfare, 2011), while in the US, only $49 \%$ of infants are breastfed to 6 months of age (Centers for Disease Control and Prevention, 2013). In Hong Kong, breastfeeding attrition is even higher with $63 \%$ of breastfeeding mothers still breastfeeding at one month, $47 \%$ at two months, and only $27 \%$ still giving any breastmilk at six months (Tarrant et al., 2010).

The major reasons for premature weaning are breastfeeding problems, rather than simply maternal choice (Meedya et al., 2010, Taveras et al., 2003, Thulier and Mercer, 2009). Women who discontinue breastfeeding prematurely are often reluctant to seek help from others when problems arise as they perceive that breastfeeding is a simple and natural task that they should be able to master by themselves (Hegney et al., 2008). The high rate of 
breastfeeding cessation in Hong Kong has been attributed to hospital practices that do not adequately support breastfeeding women (Bai et al., 2013, Parry et al., 2013, Tarrant et al., 2011), sociocultural variables and a lack of family and community support for breastfeeding (Dodgson et al., 2003, Ku and Chow, 2010, Leung et al., 2002, Tarrant et al., 2002). In addition, approximately $75 \%$ of Hong Kong women $25-45$ years of age work fulltime (Census and Statistics Department, 2011) and the maximum maternity leave available is 10 weeks with a minimum of two weeks to be taken before the expected date of confinement (Hong Kong Labour Relations Promotion Unit, 2013). Thus most women return to work at eight weeks postpartum and employers rarely provide specific support for working breastfeeding mothers (Tarrant et al., 2002).

Traditional Chinese postpartum practices and family dynamics also play a significant role in Hong Kong women's breastfeeding practices. Chinese mothers are recommended to spend the first month after birth resting and recovering at home and observing a set of rituals collectively known as "doing the month" (Pillsbury, 1982). To aid in recovery and to bring the body back to a normal and balanced state, new mothers must eat certain foods, such as chicken, Chinese ginger, and eggs, and avoid certain activities such as bathing, washing their hair, and going outside (Leung et al., 2005). At this time, the position of the mother-in-law is very dominant and most new mothers are expected to accede to their mother-in-law's recommendations and guidance, especially with regard to the care of the newborn and even with respect to breastfeeding or formula feeding (Tarrant et al., 2004). The immediate postnatal period can be isolating for the new mother as she is often cut off from her friends and is often under the direct control of her mother-in-law. Thus, when new mothers encounter breastfeeding problems they are often pressured by their mother-in-law to supplement with or switch to infant formula (Tarrant et al., 2002).

Our understanding of the factors that affect early breastfeeding cessation in this and other populations, has primarily been gained from quantitative studies (Baxter et al., 2009, 
Brown and Jordan, 2013, Gerd et al., 2012, Hauck et al., 2011). These studies have identified maternal characteristics (young maternal age, low maternal education, primiparas, and maternal smoking) birth complications (caesarean section, failure to progress), hospital practices (pacifier use, formula supplementation) and breastfeeding problems (poor sucking, poor weight gain) as contributing to early breastfeeding cessation. Only a few recent studies have explored this issue with new mothers using in-depth exploratory designs (Bailey et al., 2004, Hinsliff-Smith et al., 2014, Sheehan et al., 2009, Williamson et al., 2011). New mothers interviewed by Bailey et al. (2004) reported that they expected breastfeeding to be difficult and they felt more familiar and confident in their ability to formula feed. In contrast mothers interviewed by Williamson et al. (2011) felt a disconnect between what they had perceived as a natural and easy task with the reality of the breastfeeding difficulties that they faced.

Breastfeeding cessation is a complex and multi-factorial behaviour that cannot be adequately explored in a quantitative study (Sheehan et al., 2010) and the exclusive use of quantitative methodologies to study such a complex issue oversimplifies and misses some key nuances of the breastfeeding decision-making process (Hofvander, 2003). In order to increase breastfeeding rates and improve the health of infants, it is important to further understand why women stop breastfeeding. The purpose of this research was to explore the breastfeeding experiences of Hong Kong women who stop breastfeeding in the first month postpartum in order to identify contributing factors that might be remediated to help women breastfeed longer.

\section{Methods}

\section{Design and participants}

We used a qualitative design to explore the breastfeeding experiences of a sample of Hong Kong mothers $(\mathrm{n}=24)$ who were participants in a larger longitudinal breastfeeding 
cohort study $(\mathrm{n}=1417)$ conducted in four tertiary-care public hospitals in Hong Kong (Tarrant et al., 2010). Exploratory designs are best when the researcher is seeking to better understand an experience or aspect of life that is currently not well understood or is under researched (Hesse-Biber and Leavy, 2010). Only healthy, full-term mother-infant dyads were recruited into the larger cohort study. Specific selection criteria for the larger study were: intention to breastfeed, singleton pregnancy, Cantonese speaking, Hong Kong residents, and no serious medical complications (e.g., heart diseases, psychiatric illness, autoimmune disorders, etc.). Participants were excluded from the study if their baby was born at $<37$ weeks gestation, had a birth weight $<2500$ gm., was admitted to the neonatal intensive care unit after birth, or was born with any severe medical conditions or congenital malformations. For this part of the study we selected a subsample of participants who had breastfed for less than four weeks. Participants who met this criterion were contacted and invited to participate in a qualitative interview. A total of 371 participants were eligible for the qualitative interview. Of these 371 , we were able to contact 73 on the first attempt and 31 agreed to participate. Subsequently, seven participants were unable to participate because of scheduling problems or unexpected events, leaving a final sample of 24 mothers. After 24 interviews, data saturation was achieved and further sampling was not required.

\section{Research setting}

Hong Kong is one of the most densely populated cities in the world, with $95 \%$ of its population ethnically Chinese (Census and Statistics Department, 2011). Antenatal, postnatal, and well-child health care is provided free to all Hong Kong women through a comprehensive network of 32 Maternal and Child Health Centres (MCHCs) and eight publicly funded tertiary-care hospitals. Consequently, mothers and babies in Hong Kong have some of the lowest maternal and infant mortality rates in the world (Kwan and Leung, 2011). Although there are also nine private hospitals providing obstetric services, in 2011 public hospitals accounted for $67.8 \%$ of all births to Hong Kong mothers (Panel on Health Services, 2012). 
Breastfeeding is actively promoted through the antenatal clinics and public hospitals and after hospital discharge, postnatal breastfeeding support is available through the MCHCs. The Hong Kong Department of Health breastfeeding recommendations (Department of Health, 2011) are consistent with those of the WHO (World Health Organization, 2003), in that they recommend exclusive breastfeeding for the first six months with continued breastfeeding along with appropriate complementary feeding for up to two years and beyond. The normal length of hospital stay for uncomplicated vaginal birth is around 48 hours and for caesarean section is 72 hours and the postnatal care provided is similar to that in most large tertiary care hospitals in developed countries. Although almost all nurses working in peripartum and postnatal settings in Hong Kong public hospitals are Registered Midwives, public hospitals are busy and there is often not enough time for individualized breastfeeding support. Thus, formula supplementation is common (Parry et al., 2013). The four study hospitals each had over 300 births per month and were geographically distributed around Hong Kong.

\section{Data collection}

Semi-structured, in-depth interviews were conducted at the participants' convenience and at a location suitable to them. In most cases $(n=20)$, this was in the participant's home. Three interviews were conducted in an interview room at the School of Nursing, and one interview was conducted in a small outdoor park near the participant's home. All interviews were conducted in private, with only the participant, the infant, the interviewer and the translator present. Semi-structured interviews were the most appropriate data collection method for this study as the researcher wanted to focus on a specific research interest, early breastfeeding cessation (Kelly, 2010). An interview guide with open-ended questions was developed for the study. Interview questions focused on the participants' breastfeeding experiences prior to stopping breastfeeding, their decision-making process, the impact of hospital practices on their breastfeeding experience, post-partum support including the influences of their spouse and significant family members on their breastfeeding practices, 
societal influences, and work influences. Fourteen interviews were conducted completely in Cantonese using a translator, seven were conducted in English without the use of a translator, and three were conducted mainly in English but with the translator present to assist when necessary. Interviews lasted from 45 to 90 minutes and were digitally recorded and transcribed verbatim.

Interviews were transcribed using a process of 'naturalism,' whereby all speech patterns (i.e., pauses, laughter, vocalizations) were transcribed exactly as they were in the interview (Oliver et al., 2005). This was done to ensure the accuracy of the transcription and to avoid the transcriptionist making any changes in what participants said. However, to simplify the presentation of the textual data, the selected quotes were presented using 'denaturalism" where the vocalizations are removed and grammar is corrected so that the text reads smoothly without changing the underlying meaning of the participant's words or the substance of the interview (Oliver et al., 2005). Either the first author (MT) or third author (KW) verified the accuracy of each translated interview, depending on the language of the interview.

\section{Data analysis}

Prior to data analysis, all transcripts were read through several times to allow the researchers to become immersed in the data. A content analysis procedure was used with open coding, to facilitate the emergence of participants' voices and to avoid any preconceived notions from affecting the analysis (Krippendorff, 2012). All text was assigned codes to capture a complete picture of participants thoughts (Liamputtong, 2009). Once all data were coded, similar codes were grouped together into categories and then further abstracted into larger core themes (Green and Thorogood, 2009). Once the thematic structure was identified, the transcripts were reviewed again to validate the trustworthiness and the completeness of the data analysis process (Miles et al., 2013). In addition, the participants own words are used to substantiate the authors' analysis and interpretation of the data. Throughout data analysis, 
the NVIVO 9.0 software program (QSR International, Doncaster, Victoria, Australia) was used to assist with organization, management, and analysis of the data (QSR International, 2010).

\section{Ethical considerations}

For the larger study, ethical approval was obtained from the Institutional Review Board of the University of Hong Kong/Hospital Authority Hong Kong West Cluster and all participating institutions and informed written consent was obtained from all participants. Specific ethical approval was obtained to conduct this aspect of the study and an additional informed written consent was obtained from all participants in the qualitative interviews. As part of the consent process, participants were informed that they were free to withdraw from the study at any time, that they were free to refuse to answer any question they did not want to answer, that information divulged in the interviews would be kept in the strictest confidence, that their name would not be associated with any information they provided, that only anonymous study data would be analysed, published or presented to outside parties, and that their identity would not be made available to anyone other than the research team.

\section{Findings}

The demographic characteristics of participants are summarised in Table 1. Participants were all married and the majority (93.3\%) were above 25 years of age. Eight participants had previous children, six of whom had some previous breastfeeding experience. One participant had breastfed the preceding child for 14 weeks, two had breastfed for 4 weeks, and three had breastfed for 2 weeks. The mean duration of breastfeeding with the current child was 2.4 weeks $(\mathrm{SD}=0.50)$ with a range from 2 weeks to 3.5 weeks. Threequarters of participants had attended an antenatal childbirth preparation class and two-thirds had attended a specific breastfeeding class. Most participants (87.5\%) returned to work full- 
time after maternity leave at 8 weeks postpartum. Following data analysis, five core themes emerged that described the perceptions of the participants over the course of their short breastfeeding experience: (1) unnatural expectations; (2) left to 'figure it out'; (3) uncertainty; (4) unfulfilling experiences; and (5) guilt vs. relief.

\section{Unnatural expectations}

Hong Kong mothers, as do new mothers in many countries, believe that because breastfeeding is "natural" this also means that it will be easy. The expectation that breastfeeding would come "naturally" to both mother and infant was a common perception among mothers in this study. Although most mothers had attended some form of childbirth or breastfeeding classes in the antenatal period, none felt adequately prepared for their actual breastfeeding experience.

"I thought when the baby was born, the breast milk would come out naturally and the baby would just put her mouth to the nipple, then she could be fed. But it's totally a different thing. . . . I didn't know how to feed my baby [Participant \#14]."

Participants felt that antenatal classes primarily provided information on the advantages of breastfeeding, much of which they already knew, but little about the actual breastfeeding experience. Some participants stated that it might have been helpful to have experienced mothers come and share their breastfeeding experiences with pregnant women to better prepare them for the reality of breastfeeding.

"It was much more difficult than I thought. . . After I watched the video, I told myself that it is very easy to do. You think it's natural. Everyone can do it. But it's quite different [Participant \#16]."

Overall, participants expressed frustration that they were so inadequately prepared and that if they had known breastfeeding could be so difficult, especially in the beginning, they may have been better prepared. However, as they were expecting breastfeeding to be easy and natural, they were surprised at the difficulties that they faced. 


\section{Left to 'figure it out'}

Many mothers reported that because the midwives in the public hospitals were just too busy to provide much direct breastfeeding support, they were left to just figure out how to breastfeed on their own. After birth it was up to the mothers to know how to position the baby and to get the baby to latch on to the breast and to assess whether the baby was feeding adequately or not.

They didn't teach me. They just gave the baby to me after six hours, and then said nothing. They just said, "You can try." . . Because the nurse was quite busy [Participant \#20].

Because of insufficient nurses, they always asked me to try by myself when I asked for help. I couldn't help because after I tried, I didn't know what to do.

I know the Hong Kong government really wants to promote breastfeeding [Participant \#22].

Participants did not express anger toward the midwives on the obstetric wards for not helping them with breastfeeding, as they largely understood that maternity wards in public hospitals were very short of staff and midwives were just too busy to be able to help. Nonetheless, participants did feel abandoned and left completely on their own.

Hong Kong does not have routine postnatal home visits for new mothers as happens in some other countries like the UK and Canada. Breastfeeding mothers who need support or assistance after hospital discharge must travel to the nearest MCHC. While some mothers did report going to the $\mathrm{MCHC}$, most did not. Participants who did go, found the MCHC midwives helpful, but then felt lost again when they went back home because few had family members who could provide adequate emotional or informational support for breastfeeding.

My family did not help me. My husband could only pass me the bottle and the towel; he could do nothing else. They did not really care about whether I could breastfeed or not because they were mainly focused on the baby. Also, my mother-in-law did not breastfeed herself. When I did not have enough breastmilk or the baby cried, she would ask me to give the baby formula milk. So there was not much support from the family [Participant \#6].

Some participants expressed frustration at the contradiction in the Hong Kong government's position on breastfeeding. Breastfeeding was actively promoted in the publicly 
funded antenatal clinics and all participants were strongly encouraged to breastfeed. However, participants were frustrated that the government actively promotes breastfeeding but does not put many resources into supporting breastfeeding mothers in the immediate postnatal period. Many mothers suggested that midwives should provide home visits in the immediate postnatal period as they do in many other countries.

But basically, there is no one to support me no matter how the government promotes it to us... Something more should be done. It would be very good to have a nurse coming to our homes although I know it's difficult to do that due to the shortage of nurses. But I think it's a good idea [Participant \#8].

\section{Uncertainty}

New mothers had difficulty in gauging whether or not the infant was getting adequate nutrition from their breastmilk. Most expressed a strong need to know how much milk their newborn was getting and many held the misconception that the baby needed the same volume of breastmilk as he would formula. Because the mothers received so little support in the immediate post-natal period, they had many doubts and uncertainties about their ability to breastfeed their newborn. In most cases, the only way to resolve the uncertainty involved in breastfeeding was to provide the infant with formula milk. This reassured the participants that the baby was getting adequate nutrition and was not starving.

"I was very tired. I had no experience. There was no one to help me. I don't know how much the baby had taken. I don't know if it is okay after feeding. The key point is I didn't know whether my baby was drinking or not [Participant \#15]."

"There was a problem, as my breasts are not a feeding bottle or cup, I could see my baby's sucking but I didn't know how much my baby had taken. I doubted because I didn't know if my baby was full or not. Because the nurse told me that sucking didn't mean drinking [Participant \#13]."

In addition, significant others were often more confortable with formula feeding because it was measurable and thus easier to know if the baby had enough to drink. Many mothers reported that their husband and/or mother-in-law preferred them to 'top-up' breastfeeding with infant formula to make sure the baby was sufficiently nourished. 
What they were concerned with the most was if the baby was full or not. They worried that the baby might not get enough food. They didn't ask me not to breastfeed. They asked me to supplement with formula milk after breastfeeding [Participant \#18].

\section{Unfulfilling experiences}

Few participants had positive breastfeeding experiences and most found the experience extremely stressful and difficult. Participants described struggling during the first few days trying to breastfeed but not being able to do so because the newborn did not want to suck or they did not think they had enough milk to adequately feed the baby. Many babies cried a lot and were not content and this made the mothers constantly worried, anxious and highly stressed. From the beginning, breastfeeding was physically and emotionally challenging as most participants were exhausted, overwhelmed and receiving conflicting advice from both family and health-care professionals.

It was very difficult and I felt a lot of pain. My milk wasn't enough and he drank a lot. He seemed to be still hungry even if I had breastfed him for a long time. The nurse asked me if this was the second time feeding the baby. And I said I was still feeding him - I fed him continuously. So the nurse took the baby and gave him bottle milk in order to let me take a rest [Participant \#4].

After returning home from the hospital, participants struggled to continue breastfeeding but were never able to overcome the difficulties to establish nursing and enjoy that time with their babies.

"I wanted to breastfeed so much at the time, but I had insufficient milk. And I had great pressure. My mother-in-law cared about me, but her caring gave me pressure. She asked me why I did not breastfeed my baby. But it wasn't that I did not want to breastfeed, it was just that my baby was not able to suck. I did try for many times, but in vain [Participant \#19]."

Mothers also wondered why they could not successfully breastfeed when they knew others who had and whether or not there was something 'different' about either them or their baby that they were unable to do it.

Guilt vs. relief

The difficult decision to stop breastfeeding caused sadness and guilt for most of the participants. Some women felt that breastfeeding was intricately linked to their mothering 
abilities and if they weren't able to adequately feed their baby with their own milk then this must mean that they were an inadequate mother.

"I cried because I thought I was useless. I cried because I was not able to feed her. I wondered why it was so hard to be a mother. It affected my emotion. . . I It's a bit funny when I talk about it now because I think that I was a bit crazy at that time. I couldn't control my emotions and I cried a lot [Participant \#24]."

Despite their breastfeeding problems, some participants wanted to continue breastfeeding and felt sad and depressed when they stopped.

When I stopped, I felt empty and could not help it. It felt like something was missing. I didn't know why. I was a bit down at that time [Participant \#11].

Other participants, however, expressed relief after stopping breastfeeding because of the stress and difficulties they experienced while breastfeeding. The fact that others could now help care for the baby was a burden lifted off them.

"I felt more comfortable because I didn't have to feed every two hours and other people could help me to feed the baby. Because when I breastfed, I had to wake up and I couldn't ask others to help. But for formula feeding, others can check the time for feeding, put the milk into the bottle and then they can feed the baby. That's it. I didn't get upset. I felt better not having to breastfeed [Participant \#23]."

The frequency of breast-feeding was high because I had to feed the baby whenever she cried. I felt more comfortable after I changed to feed with formula milk because she's full and she cried less often [Participant \#1].

In addition, for mothers whose babies were not feeding well and who were not gaining

weight, the introduction of formula and the discontinuation of breastfeeding usually meant

that their babies started to gain weight quickly and also slept longer.

I breastfed exclusively and he was very thin. I was very worried because he weighed 7.5 pounds when he was born. But then he only weighed a bit more than six pounds.

So I was afraid. ... He cried because he's not getting enough. So I used formula milk as supplement. After I used formula milk as supplement, his weighted increased greatly [Participant \#9].

For others there was a mix of emotions. On one hand they felt relief that they now knew the baby was getting adequate nutrition and was gaining weight but they also felt sad and guilty that they could not give the baby their own breastmilk. 
On one hand, I felt sad because I was not able to do that. On the other hand, I had no choice because my baby had to eat. Because my baby had a big appetite at that time, I had to feed with formula milk. Afterwards I felt more relaxed because my baby could take more food. But I felt sad as well because I was not able to give him my breastmilk [Participant \#2].

\section{Discussion}

The analysis presented in this paper represents the accounts of a purposeful sample of mothers who stopped breastfeeding before 4 weeks postpartum. Study findings highlight the difficulties faced by new mothers in breastfeeding their newborn babies. Participants did not feel adequately prepared for their breastfeeding experience and did not receive sufficient support during this crucial period. Thus the breastfeeding experience was stressful and unfulfilling for participants, many of who experienced substantial guilt and self-doubt about being unable to successfully breastfeed.

Other researchers have noted that many new mothers often believe that because breastfeeding is "natural" this also means that it will be easy (Graffy and Taylor, 2005, Hauck et al., 2011, Mozingo et al., 2000, Razurel et al., 2011, Scott and Mostyn, 2003, Shakespeare et al., 2004). Williamson et al. (2011), in their phenomenological study of eight first-time mothers in the UK, found that mothers were not prepared for the breastfeeding difficulties that they would face in the first week as they expected it to be natural and innate. Redshaw \& Henderson (2012) qualitatively analysed the open-ended written responses of 2,054 participants in a large UK study on new mothers expectations of and experiences with early postnatal breastfeeding support. The authors also found that many new mothers feel unsupported and isolated in the early postpartum period and their inability to master what is supposed to be a 'natural' task causes them great distress (Redshaw and Henderson, 2012). Using a grounded theory approach, Sheehan et al., (2009) interviewed 37 Australian about their experiences with breastfeeding support in the first 6 weeks postpartum. Mothers in this study perceived that antenatal preparation for breastfeeding was inadequate and the act of 
breastfeeding was often presented as straightforward, pain free and easy to master (Sheehan et al., 2009). Healthcare professionals also perceive that many new mothers have unrealistic expectations about breastfeeding and are ill equipped for the realities they will face (Brown et al., 2011, Martin et al., 2013). Providing more realistic information to pregnant women in the antenatal period may help to better prepare them for their early postpartum breastfeeding experience. Although most participants had attended childbirth preparation and breastfeeding classes, they felt that the content of these classes was not overly helpful and did not adequately prepare them. This is consistent with the findings of a previous study on Hong Kong mothers' perceptions of antenatal classes. Ho and Holroyd (2002) reported that mothers found the information presented in the classes useful but did not feel the classes adequately prepare them for their mothering experience, especially with respect to breastfeeding. In addition, class sizes were large and the teaching mode was didactic (Ho and Holroyd, 2002), an arrangement which generally persists today.

Other studies have shown that mothers who persist at breastfeeding do anticipate that it will be difficult at first but they have more commitment and determination to overcome the problems encountered (Avery et al., 2009, Tarrant et al., 2004). Although many new mothers expect breastfeeding to happen naturally and instinctively, in many developed countries bottle-feeding is more the cultural norm and breastfeeding is largely hidden away (Blum, 1999, Scott and Mostyn, 2003). Infant formula manufacturers' aggressive marketing of their products over the past decades has been highly successful in creating a social preference for infant formula over breastfeeding (Kaplan and Graff, 2008), whereby formula feeding is perceived as the normal infant feeding method and breastfeeding is the deluxe or premium method (Bartick et al., 2006). In Hong Kong, less than one-half of young adults surveyed reported that they had ever witnessed a women breastfeeding and almost $85 \%$ perceived that breastfeeding in public would be embarrassing (Tarrant and Dodgson, 2007). Conversely, in non-industrialized societies where artificial feeding is rare, breastfeeding is openly practiced, 
infants have constant access to the mother's breast, and nursing often extends well into childhood (Nelson et al., 2000). Locke (2009) recommends that instead of presenting breastfeeding as something automatic like walking or breathing, it needs to be constructed as a natural 'process' that also needs to be taught and learned.

Further antenatal and postnatal education on the issues surrounding the normal amount of breastmilk newborns require is also important. Perceived insufficient milk is one of the most frequently cited reasons for both early breastfeeding cessation (Ahluwalia et al., 2005, Hauck et al., 2011) and the early introduction of infant formula (Gagnon et al., 2005). Because of the success of infant formula manufacturers in creating a bottle-feeding culture, many new mothers have expectations about the amount of milk that newborns should take (Whitaker and Wright, 2012) and many hold the misconception that the volume of breastmilk and formula required are equal (American Academy of Pediatrics, 2012). Similarly, HinsliffSmith et al. (2014) found that many new mothers assumed that the volume of their breastmilk would be directly comparable to the volume of infant formula being offered in postnatal wards and that the breastmilk would come 'gushing' out after birth.

The lack of in-hospital support for breastfeeding mothers and the sense of abandonment that many mothers report after leaving the hospital are also informative for health policy and hospital practices. When breastfeeding problems arise, new mothers feel that there is no one to rely on for help and support. Studies across many cultures and geographic regions show that in-hospital postnatal support for breastfeeding mothers is inadequate and not able to meet their needs (McInnes and Chambers, 2008). Once mothers are discharged home, the support is also lacking. Hong Kong, unlike other countries such as the UK and Canada, does not have public health nurses, midwives or health visitors conducting home visits to new mothers. Upon hospital discharge, mothers are advised to visit the nearest $\mathrm{MCHC}$ within the first week. However, the early postpartum period can be overwhelming for new mothers and it may be difficult for them to actively seek support themselves. In addition, 
going outside the home in the early postpartum period is strongly discouraged as it violates the recommendations of doing-the-month (Pillsbury, 1982), thus further reducing the likelihood of new mothers seeking breastfeeding support. For government public health campaigns to promote breastfeeding to truly be successful, adequate infrastructure to support these policies need to be in place (United States Department of Health \& Human Services, 2011) and need to be consistent with local norms and cultural practices.

In Hong Kong, when breastfeeding problems arise many new mothers face substantial family and sociocultural pressure to stop breastfeeding and supplement with infant formula (Tarrant et al., 2004). Participants in other studies who persevered with breastfeeding have identified early professional support as one of the most important determinants of whether they stopped or continued breastfeeding (Kelleher, 2006). Private lactation and postnatal midwifery support services are available from independently employed Lactation Consultants and midwives, but only to those who can afford it. Also, mothers who stopped breastfeeding early reported that they might have been able to continue if they had received adequate professional support (Redshaw and Henderson, 2012). This has implications for public health policy and planning in Hong Kong and elsewhere.

Although midwives do provide a substantial amount of the antenatal, intrapartum, and postnatal care for childbearing women in Hong Kong, the overall approach is a shared-care model whereby obstetricians, midwives, and nurses play various roles and the organization of care is compartmentalized accordingly (Sandall et al., 2009). Pregnant women do not have an assigned midwife and the philosophy of care is not consistent with a midwifery-led model. A recent review of 13 studies with $>16,000$ women evaluating different models of maternity care however, has found that when compared with care models such as shared care or obstetrician-led care, having a midwife as the main care provider substantially improves many pregnancy outcomes (Sandall et al., 2013). 
Although some participants expressed relief after stopping breastfeeding, few had positive breastfeeding experiences and many were left feeling inadequate as mothers because of their failure to breastfeed. Kelleher (2006) also reported that women who stopped breastfeeding early expressed feelings of guilt and personal failure at their inability to breastfeed. This creates a situation where her doubts about her mothering ability could continue to affect her relationship with her child beyond infancy, an important topic for further study. Among the participants, although six had previously breastfed, only one participant had breastfed for longer than four weeks. Breastfeeding experiences with a first child are likely to be repeated with second and subsequent children (Kruse et al., 2006, Phillips et al., 2011) and thus mothers with previously unsuccessful breastfeeding experiences are at high risk for a short duration of breastfeeding with future children. If lactation support resources are limited this group should be targeted.

\section{Limitations}

This study relates the breastfeeding experiences of 24 women with a short duration of breastfeeding. Thus, the findings represent the experiences of a small proportion of breastfeeding women who stop breastfeeding early and the generalisability of the findings to the larger population of breastfeeding Chinese mothers should be done with caution. Furthermore, in qualitative studies it is necessary to aggregate data in a manner that may oversimplify individual participant's experiences. Each participant's breastfeeding experience was multifaceted and complex and it is possible that that it was not adequately represented in the study results. Finally, many of the interviews were conducted in Chinese and the interviewer (MT) relied on a translator to report the participants' dialogue. Translation errors and miscommunication many have occurred during the interviews, which may have resulted in misinterpretation of the participants' responses. However, an experienced and fluently bilingual translator was used and thus the researchers believe that the issue of misinterpretation is likely minimal. 


\section{Conclusion}

Our findings highlight the importance of early one-to-one support with initiation of breastfeeding after birth and on-going psychological and emotional support after hospital discharge. Further antenatal and postnatal education about the issues surrounding the amount of breastmilk taken in by babies is also important to counter misperceptions about insufficient milk and the need to supplement with infant formula. Greater postnatal breastfeeding support, both in the hospital and after the mother returns home, would likely increase the mother's confidence in breastfeeding and enhance her mothering experience. Further research on breastfeeding support interventions in the early postnatal period would help to identify effective strategies to prolong the duration of breastfeeding and to reduce early formula supplementation. All of these findings indicate that the existing health care system is not meeting the needs of many postpartum mothers, especially with respect to breastfeeding. Further breastfeeding support is needed using a multi-pronged approach at both the healthcare service and the health policy levels. 


\section{References}

Ahluwalia, I.B., Morrow, B., Hsia, J., 2005. Why do women stop breastfeeding? Findings from the Pregnancy Risk Assessment and Monitoring System. Pediatrics 116 (6), 1408-1412.

American Academy of Pediatrics, 2012. Policy statement: Breastfeeding and the use of human milk. Pediatrics 129 (3), e827-841.

Australian Institute of Health and Welfare, 2011. 2010 Australian National Infant Feeding Survey: indicator results. AIHW, Canberra.

Avery, A., Zimmermann, K., Underwood, P.W., Magnus, J.H., 2009. Confident commitment is a key factor for sustained breastfeeding. Birth 36 (2), 141-148.

Baby Friendly Hospital Initiative Hong Kong Association, 2013. World breastfeeding week 2013: annual summary. Author, Hong Kong.

Bai, D.L., Wu, K.M., Tarrant, M., 2013. Association between intrapartum interventions and breastfeeding duration. Journal of Midwifery \& Women's Health 58 (1), 25-32.

Bailey, C., Pain, R.H.R.H., Aarvold, J.E.J.E., 2004. A 'give it a go' breast-feeding culture and early cessation among low-income mothers. Midwifery 20 (3), 240-250.

Bartick, M.C., Walker, M., Bagley, D.A., Wiessinger, D., 2006. Breastfeeding as normal: an urban breastfeeding advertising campaign inspired by the tobacco industry. In: American Public Health Association's 134th Annual Meeting - Public Health and Human Rights. Boston, MA.

Baxter, J., Cooklin, A.R., Smith, J., 2009. Which mothers wean their babies prematurely from full breastfeeding? An Australian cohort study. Acta Paediatrica 98 (8), 1274-1277.

Blum, L.M., 1999. At the breast: Ideologies of breastfeeding and motherhood in the contemporary United States. Beacon Press, Boston, MA.

Brown, A., Jordan, S., 2013. Impact of birth complications on breastfeeding duration: an internet survey. Journal of Advanced Nursing 69 (4), 828-839.

Brown, A., Raynor, P., Lee, M., 2011. Healthcare professionals' and mothers' perceptions of factors that influence decisions to breastfeed or formula feed infants: a comparative study. Journal of Advanced Nursing 67 (9), 1993-2003.

Cattaneo, A., Burmaz, T., Arendt, M., Nilsson, I., Mikiel-Kostyra, K., Kondrate, I., Communal, M.J., Massart, C., Chapin, E., Fallon, M., 2010. Protection, promotion and support of breast-feeding in Europe: progress from 2002 to 2007. Public Health Nutrition 13 (6), 751-759. 
Census and Statistics Department, 2011. Women and men in Hong Kong: key statistics. 2011 edition. Hong Kong Census and Statistics Department, Hong Kong.

Centers for Disease Control and Prevention, 2013. Breastfeeding Report Card-United States, 2013. Author, Atlanta.

Department of Health, 2011. DH committed to promoting breastfeeding. Centre for Health Protection, Hong Kong.

Department of Health, 2013. Indicators on Breastfeeding: Quarter 4 2012/13. Health Improvement Analytical Team London.

Dodgson, J.E., Tarrant, M., Fong, D.Y.T., Peng, X.H., Hui Choi, E.W.H., 2003. Breastfeeding patterns of primiparous mothers in Hong Kong. Birth 30 (3), 195-202.

Gagnon, A.J., Leduc, G., Waghorn, K., Yang, H., Platt, R.W., 2005. In-hospital formula supplementation of healthy breastfeeding newborns. Journal of Human Lactation 21 (4), 397-405.

Gerd, A.-T., Bergman, S., Dahlgren, J., Roswall, J., Alm, B., 2012. Factors associated with discontinuation of breastfeeding before 1 month of age. Acta Paediatrica 101 (1), 5560.

Graffy, J., Taylor, J., 2005. What information, advice, and support do women want with breastfeeding? Birth 32 (3), 179-186.

Green, J., Thorogood, N., 2009. Qualitative methods for health research. SAGE, Los Angeles.

Guo, S., Fu, X., Scherpbier, R.W., Wang, Y., Zhou, H., Wang, X., Hipgrave, D.B., 2013. Breastfeeding rates in central and western China in 2010: implications for child and population health. Bulletin of the World Health Organization 91 (5), 322-331.

Hauck, Y.L., Fenwick, J., Dhaliwal, S.S., Butt, J., 2011. A Western Australian survey of breastfeeding initiation, prevalence and early cessation patterns. Maternal \& Child Health Journal 15 (2), 260-268.

Hauck, Y.L., Graham-Smith, C., McInerney, J., Kay, S., 2011. Western Australian women's perceptions of conflicting advice around breast feeding. Midwifery 27 (5), 156-162.

Hegney, D., Fallon, T., O'Brien, M.L., 2008. Against all odds: a retrospective case-controlled study of women who experienced extraordinary breastfeeding problems. Journal of Clinical Nursing 17 (9), 1182-1192.

Hesse-Biber, S.J.N., Leavy, P.L., 2010. The practice of qualitative research. Sage, Thousand Oaks, CA. 
Hinsliff-Smith, K., Spencer, R., Walsh, D., 2014. Realities, difficulties, and outcomes for mothers choosing to breastfeed: Primigravid mothers experiences in the early postpartum period (6-8 weeks). Midwifery 30 (1), e14-19.

Ho, I., Holroyd, E., 2002. Chinese women's perceptions of the effectiveness of antenatal education in the preparation for motherhood. Journal of Advanced Nursing 38 (1), 7485.

Hofvander, Y., 2003. Why women don't breastfeed: a national survey. Acta Paediatrica 92 (11), 1243-1244.

Hong Kong Labour Relations Promotion Unit, 2013. The Employment Ordinance, Cap. 57: Maternity protection. The Government of the Hong Kong SAR Labour Department. Ip, S., Chung, M., Raman, G., Chew, P., Magula, N., Devine, D., Trikalinos, T., Lau, J., 2007. Breastfeeding and maternal and infant health outcomes in developed countries. Evidence Report/Technology Assessment (153), 1-186.

Kaplan, D.L., Graff, K.M., 2008. Marketing breastfeeding--reversing corporate influence on infant feeding practices. Journal of Urban Health 85 (4), 486-504.

Kelleher, C.M., 2006. The physical challenges of early breastfeeding. Social Science \& Medicine 63 (10), 2727-2738.

Kelly, S.E., 2010. Qualitative interviewing techniques and styles. In: Bourgeault, I., Dingwall, R., De Vries, R. (Eds.), Qualitative methods in health research. Sage, Los Angeles, CA, pp. 307-326.

Kramer, M.S., Kakuma, R., 2004. The optimal duration of exclusive breastfeeding: a systematic review. Advances in Experimental Medicine and Biology 554, 63-77.

Krippendorff, K.H., 2012. Content analysis: an introduction to its methodology. Sage, Thousand Oaks, CA.

Kruse, L., Denk, C.E., Feldman-Winter, L., Rotondo, F.M., 2006. Longitudinal patterns of breastfeeding initiation. Maternal \& Child Health Journal 10 (1), 13-18.

Ku, C.M., Chow, S.K., 2010. Factors influencing the practice of exclusive breastfeeding among Hong Kong Chinese women: a questionnaire survey. Journal of Clinical Nursing 19 (17-18), 2434-2445.

Kwan, D., Leung, E., 2011. Vital Statistics in 2010. Public Health \& Epidemiology Bulletin 20 (2), 15-30.

Leung, G.M., Ho, L.M., Lam, T.H., 2002. Breastfeeding rates in Hong Kong: a comparison of the 1987 and 1997 birth cohorts. Birth 29 (3), 162-168. 
Leung, S.K., Arthur, D., Martinson, I.M., 2005. Perceived stress and support of the Chinese postpartum ritual "doing the month". Health Care for Women International 26 (3), 212-224.

Liamputtong, P., 2009. Qualitative research methods. Oxford University Press Australia and New Zealand, South Melbourne, Vic.

Locke, A., 2009. 'Natural versus taught': competing discourses in antenatal breastfeeding workshops. Journal of Health Psychology 14 (3), 435-446.

Martin, A., Horowitz, C., Balbierz, A., Howell, E., 2013. Views of women and clinicians on postpartum preparation and recovery. Maternal and Child Health Journal, 1-7.

McInnes, R.J., Chambers, J.A., 2008. Supporting breastfeeding mothers: qualitative synthesis. Journal of Advanced Nursing 62 (4), 407-427.

Meedya, S., Fahy, K., Kable, A., 2010. Factors that positively influence breastfeeding duration to 6 months: a literature review. Women \& Birth 23 (4), 135-145.

Miles, M.B., Huberman, A.M., Saldana, J., 2013. Qualitative data analysis: a methods sourcebook. Sage, Thousand Oaks, CA.

Mozingo, J.N., Davis, M.W., Droppleman, P.G., Merideth, A., 2000. "It wasn't working": women's experiences with short-term breastfeeding. MCN, American Journal of Maternal Child Nursing 25 (3), 120-126.

Nelson, E.A., Schiefenhoevel, W., Haimerl, F., 2000. Child care practices in nonindustrialized societies. Pediatrics 105 (6), E75.

Oliver, D.G., Serovich, J.M., Mason, T.L., 2005. Constraints and opportunities with interview transcription: Towards reflection in qualitative research. Social Forces 84 (2), 1273 1289.

Organisation for Economic Co-operation and Development, 2009. OECD Family database: breastfeeding rates. In: OECD Family Database. OECD, Paris.

Panel on Health Services, 2012. Latest arrangement for non-local pregnant women giving birth in Hong Kong. Hong Kong.

Parry, J.E., Ip, D.K., Chau, P.Y., Wu, K.M., Tarrant, M., 2013. Predictors and consequences of in-hospital formula supplementation for healthy breastfeeding newborns. Journal of Human Lactation 29 (4), 527-536.

Phillips, G., Brett, K., Mendola, P., 2011. Previous breastfeeding practices and duration of exclusive breastfeeding in the United States. Maternal \& Child Health Journal 15 (8), 1210-1216. 
Pillsbury, B.L.K., 1982. 'Doing the month': confinement and convalescence of Chinese women after childbirth. In: Kay, M.A. (Ed.), Anthropology and human birth. F. A. Davis, Philadelphia, PA.

QSR International, 2010. NVIVO Software: Release 9.0. QSR International, Doncaster, Australia.

Razurel, C., Bruchon-Schweitzer, M., Dupanloup, A., Irion, O., Epiney, M., 2011. Stressful events, social support and coping strategies of primiparous women during the postpartum period: a qualitative study. Midwifery 27 (2), 237-242.

Redshaw, M., Henderson, J., 2012. Learning the hard way: expectations and experiences of infant feeding support. Birth 39 (1), 21-29.

Sandall, J., Hatem, M., Devane, D., Soltani, H., Gates, S., 2009. Discussions of findings from a Cochrane review of midwife-led versus other models of care for childbearing women: continuity, normality and safety. Midwifery 25 (1), 8-13.

Sandall, J., Soltani, H., Gates, S., Shennan, A., Devane, D., 2013. Midwife-led continuity models versus other models of care for childbearing women. Cochrane Database of Systematic Reviews (8).

Scott, J.A., Mostyn, T., 2003. Women's experiences of breastfeeding in a bottle-feeding culture. Journal of Human Lactation 19 (3), 270-277.

Shakespeare, J., Blake, F., Garcia, J., 2004. Breast-feeding difficulties experienced by women taking part in a qualitative interview study of postnatal depression. Midwifery 20 (3), 251-260.

Sheehan, A., Schmied, V., Barclay, L., 2010. Complex decisions: theorizing women's infant feeding decisions in the first 6 weeks after birth. Journal of Advanced Nursing 66 (2), 371-380.

Sheehan, A., Schmied, V., Barclay, L., 2009. Women's experiences of infant feeding support in the first 6 weeks post-birth. Maternal \& Child Nutrition 5 (2), 138-150.

Tarrant, M., Dodgson, J.E., 2007. Knowledge, attitudes, exposure, and future intentions of Hong Kong University Students toward infant feeding. JOGNN 36 (3), 243-254.

Tarrant, M., Dodgson, J.E., Choi, V.W.K., 2004. Becoming a role model: the breastfeeding trajectory of Hong Kong women breastfeeding longer than six months. International Journal of Nursing Studies 41 (5), 535-546.

Tarrant, M., Dodgson, J.E., Tsang, S.F., 2002. Initiating and sustaining breastfeeding in Hong Kong: contextual influences on new mothers' experiences. Nursing \& Health Sciences $4(4), 189-191$. 
Tarrant, M., Fong, D.Y., Wu, K.M., Lee, I.L., Wong, E.M., Sham, A., Lam, C., Dodgson, J.E., 2010. Breastfeeding and weaning practices among Hong Kong mothers: a prospective study. BMC Pregnancy \& Childbirth 10, 27.

Tarrant, M., Wu, K.M., Fong, D.Y., Lee, I.L., Wong, E.M., Sham, A., Lam, C., Dodgson, J.E., 2011. Impact of baby-friendly hospital practices on breastfeeding in Hong Kong. Birth 38 (3), 238-245.

Taveras, E.M., Capra, A.M., Braveman, P.A., Jensvold, N.G., Escobar, G.J., Lieu, T.A., 2003. Clinician support and psychosocial risk factors associated with breastfeeding discontinuation. Pediatrics 112 (1 Pt 1), 108-115.

Thulier, D., Mercer, J., 2009. Variables associated with breastfeeding duration. JOGNN 38 (3), 259-268.

United States Department of Health \& Human Services, 2011. The Surgeon General's Call to Action to support breastfeeding.

Whitaker, R.C., Wright, J.A., 2012. Why feed breast milk from a bottle? Archives of Pediatrics and Adolescent Medicine 166 (5), 483-484.

Williamson, I., Leeming, D., Lyttle, S., Johnson, S., 2011. 'It should be the most natural thing in the world': exploring first-time mothers' breastfeeding difficulties in the UK using audio-diaries and interviews. Maternal \& Child Nutrition.

World Health Organization, 2003. Global strategy for infant and young child feeding. World Health Organization, Geneva. 